\title{
The effect of boundary roughness on dense granular flows
}

\author{
Farnaz Fazelpour ${ }^{1, *}$ and Karen E. Daniels ${ }^{1, * *}$ \\ ${ }^{1}$ Department of Physics, North Carolina State University, Raleigh, NC 27695, USA
}

\begin{abstract}
In the field of granular rheology, an important open question is to understand the influence of boundary conditions on granular flows. We perform experiments in a quasi-2D annular shear cell subject to 6 different boundaries with controlled roughness/compliance. We characterize the granular slip at the boundaries to investigate which aspects of a dense granular flow can be controlled by the choice of boundary condition. Photoelastic techniques are implemented to measure the stress fields $P(r)$ and $\tau(r)$ throughout the material. A full inverseanalysis of the fringes within each disk provides the vector force at each contact. This allows us to measure the continuum stress field by coarse-graining internal forces. We have observed that boundary roughness and compliance strongly controls the flow profile $v(r)$ and shear rate profile $\dot{\gamma}(r)$. We also observed that boundary roughness and compliance play a significant role in the pressure profile $P(r)$ and shear stress profile $\tau(r)$.
\end{abstract}

\section{Introduction}

Constitutive models can be postulated on general empirical grounds, relating the applied stress to the flow properties. For granular materials, such constitutive models are a generalization of elastic theory and viscous theory that allows for continuum modeling of a broad class of materials. In a dense granular flow, the starting point for describing a flow is to consider the dimensionless inertial number [1]:

$$
I \equiv \frac{\dot{\gamma} d}{\sqrt{P / \rho}} \text {. }
$$

which indicates flow speed ( $d$ is the particle diameter, $P$ is the pressure, $\rho$ is the particle density and $\dot{\gamma}$ is the shear rate). It is convenient to similarly quantify the applied stress (the cause of the flow) via the nondimensional stress ratio $\mu(r)$, the ratio between the local shear stress $\tau$ and the local pressure $P$ :

$$
\mu \equiv \frac{\tau}{P}
$$

While these local rheologies have been broadly successful at describing fully-developed flows, they fail to quantitatively capture heteregeneous flows [2-6]. Due to these limitations of local rheology, nonlocal rheology models [7-10] have recently been developed. These have been shown to be successful in modeling granular flows across different packing densities, particle sizes and shapes, and shear rates [11], but it remains unclear how best to predict the flow at boundaries a priori .

In order to characterize the properties of granular slip at boundaries and incorporate our observations into a nonlocal rheology model, we measure the stress field $\mu(r)$ throughout the material. For this purpose, we use photoelastic particles: these measurements work by using polarized light to detect changes in the principle stresses within

\footnotetext{
*e-mail: ffazelp@ncsu.edu

**e-mail: kdaniel@ncsu.edu

A video is available at https://doi.org/10.48448/mcgk-s 820
}

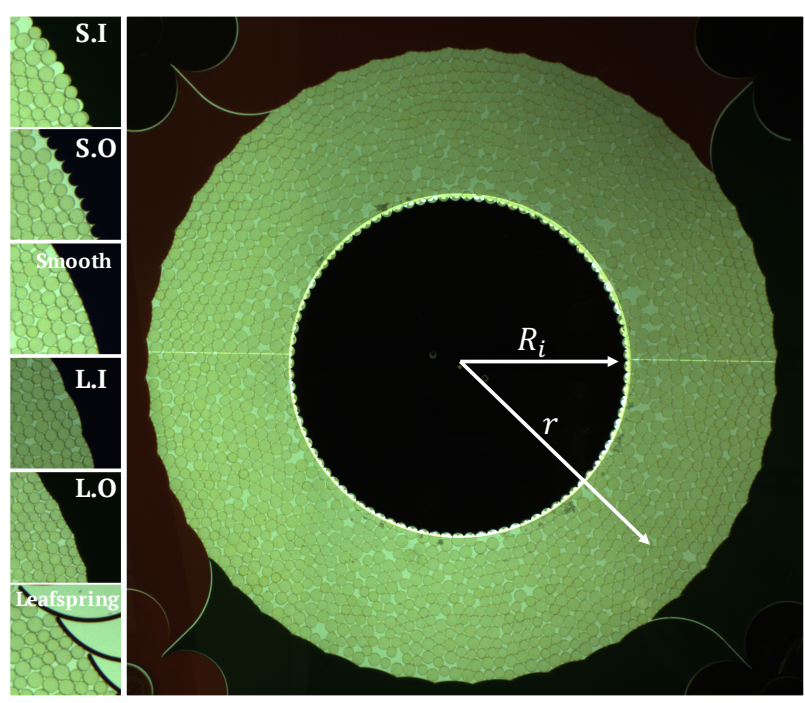

Figure 1. Top view of annular rheometer, showing five different outer wall boundary conditions. From top to bottom: small innies (to pin the flow), small outies (like a continuation of the granular material), smooth (to promote slip), large innies, large outies (to match the leaf spring shape), and flexible leaf springs.

a particle, revealing the internal forces. Photoelasticity allows us to measure a force vector at each contact by optimizing an inverse-analysis of the fringe pattern within each disk. From this discrete force data, we obtain the continuum stress field by coarse-graining. Spatial smoothing (coarse-graining) and temporal averaging enable us fully characterize the stress tensor throughout the material, beyond just boundary measurements [11]. 
a)

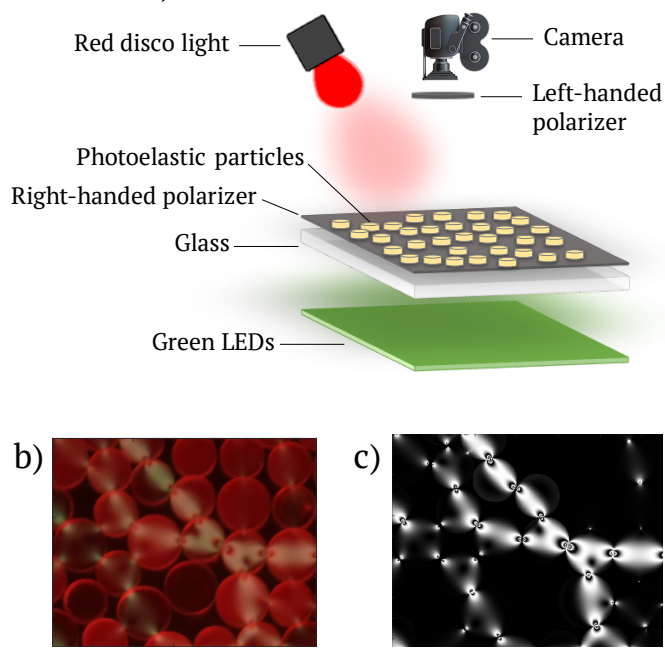

Figure 2. (a) Schematic of photoelastic setup. (b) Sample image taken with high-resolution Nikon DSLR camera. (c) The reconstructed pseudo-image, created via photoelastic inversion.

\section{Methods}

\subsection{Apparatus}

Our apparatus [11] consists of a quasi-2D annular shear cell, allowing for both continuous shearing from the inner wall $\left(R_{i}=15 \mathrm{~cm}\right)$, and visual access to the dynamics of all particles. A motor is attached to the inner wall, providing a constant rotational speed. We measure the inner wall shear stress $\tau\left(R_{i}\right)$ via a torque sensor attached to the central axle. In the experiments described below, we use $\sim 1700$ bidisperse photoelastic disks of diameter $d=9$ and $11 \mathrm{~mm}$ and thickness $6 \mathrm{~mm}$; these allow for quantitative interparticle force measurements via photoelasticity. The outer wall is stationary $\left(R_{o}=30 \mathrm{~cm}\right)$. Both walls are laser-cut and are inter-changeable to modify roughness. Fig. 1 shows closeups of these particles placed within the six different outer walls of differing roughness/compliance.

\subsection{Photoelastic measurements}

Photoelastic materials are birefringent: they rotate the polarization of incident light depending on the amount of local stress. This property reveals internal forces between particles when you place them between two circular polarizers with opposite chirality $[12,13]$. Depending on the specific vector forces on each particle, different fringe patterns arise. In our experiment, we use two sources of light: polarized green LED light transmitted through the particles, and unpolarized red disco LED light reflected off the particles (see Fig. 2(a)).

Through color separation, each image contains both particle positions (red channel) and force chains (green channel), as shown in Fig. 2(b). To measure the force vector at each contact we use the PeGS open-source analysis code [14]. In this technique, the first step is finding particle locations using the Matlab imfindcircles implementation of the Hough transform. The second step is finding possible contacts and estimating the forces by observing the gradients in each particle. The last step is performing a full inverse-analysis of the fringes by optimizing the fringe pattern within each disk, to determine the vector force at each contact. Details about this process are available in $[12,15]$, resulting in knowing the magnitude and direction of the force at each contact. An example of measurement result (pseudo-image) is shown in Fig. 2(c).

\subsection{Coarse-graining}

Next, we utilize these vector contact forces to measure the shear stress $\tau$ and pressure $P$ following the method of [16], using a Lucy function $W(r)$ to determine the stress field.

$$
W(r)= \begin{cases}\frac{5}{\pi c^{2}}\left[-3\left(\frac{r}{c}\right)^{4}+8\left(\frac{r}{c}\right)^{3}+6\left(\frac{r}{c}\right)^{2}+1\right] & |r|<c \\ 0 & |r| \geq c\end{cases}
$$

where $c$ is the coarse-graining length scale with half width $c=2 w$. We pick $w=1.3 d$ to suppress fluctuations at the particle length scale while avoiding over-smoothing the gradients in the stress field. Using $W(r)$, all components of the stress tensor $\sigma_{x x}, \sigma_{x y}, \sigma_{y x}, \sigma_{y y}$ at each point, $r$, can be derived from:

$$
\sigma \equiv \sum_{i=1}^{N} \sum_{j=i+1}^{N} f_{i j} r_{i j} \int_{0}^{1} W\left(r-r_{i}+s r_{i j}\right) d s
$$

with $f_{i j}$ the contact force between each $(i, j)$ pair of particles and $r_{i j}=r_{i}-r_{j}$ the corresponding displacement vector between the centers of the particles. Since our apparatus has an annular Couette geometry, we convert our stress measurements from cartesian to cylindrical coordinates.

Fig. 3 shows the resulting coarse-grained shear stress and pressure measurements for a sample image containing one quarter of the rheometer. The pressure is always non-negative since there is no tension force possible between particles, and shear stress can be either positive or negative depending on the direction of the shear. Since the inner disk rotates clockwise, we pick a sign convention for which clockwise shear is positive.

\section{Results}

We use these measurements to compare the flow behavior under the various boundary roughness/compliance configurations shown in Fig. 1. To explore a range of behaviors, we perform experiments under 2 different conditions. In the first series of experiments, we maintain a constant packing fraction $\phi=0.65$; these are referred to as $\phi$ constant. In the second series of experiments, we maintain a constant inner wall shear stress $\tau\left(R_{i}\right)=1960 \mathrm{~Pa}$; there are referred to as $\tau_{i}$-constant.

We have observed that the $\phi$-constant runs are not strongly affected by conditions such as temperature and humidity. However, due to the various shapes/curvatures of the walls, it is difficult to precisely measure $\phi$. Runs un$\operatorname{der} \tau_{i}$-constant conditions (adjusted by adding/subtracting 

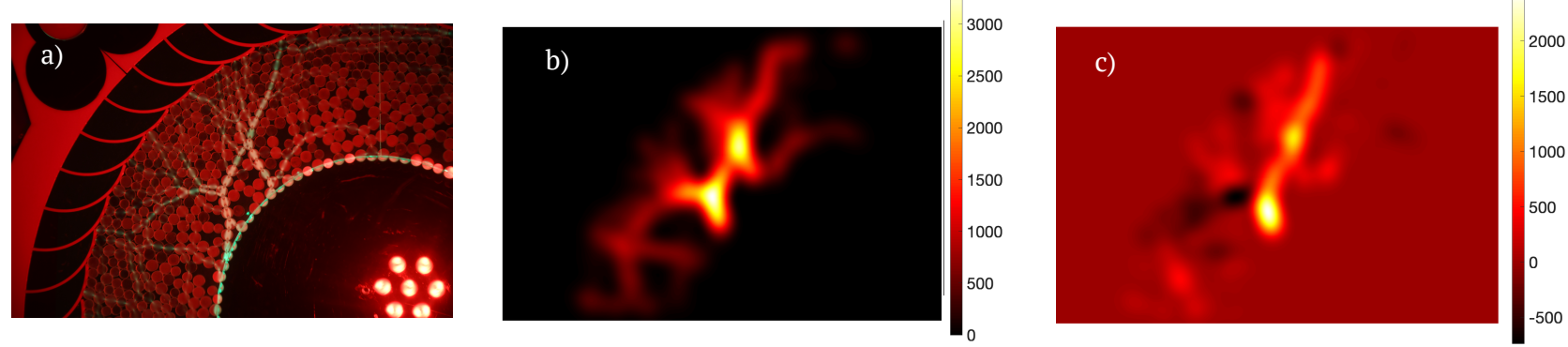

Figure 3. (a) Sample image taken with high-resolution Nikon DSLR camera. (b) Normal stress field $P(x, y)$ and (c) shear stress field $\tau(x, y)$ derived from coarse-graining. Colorbar is stress in [Pa].
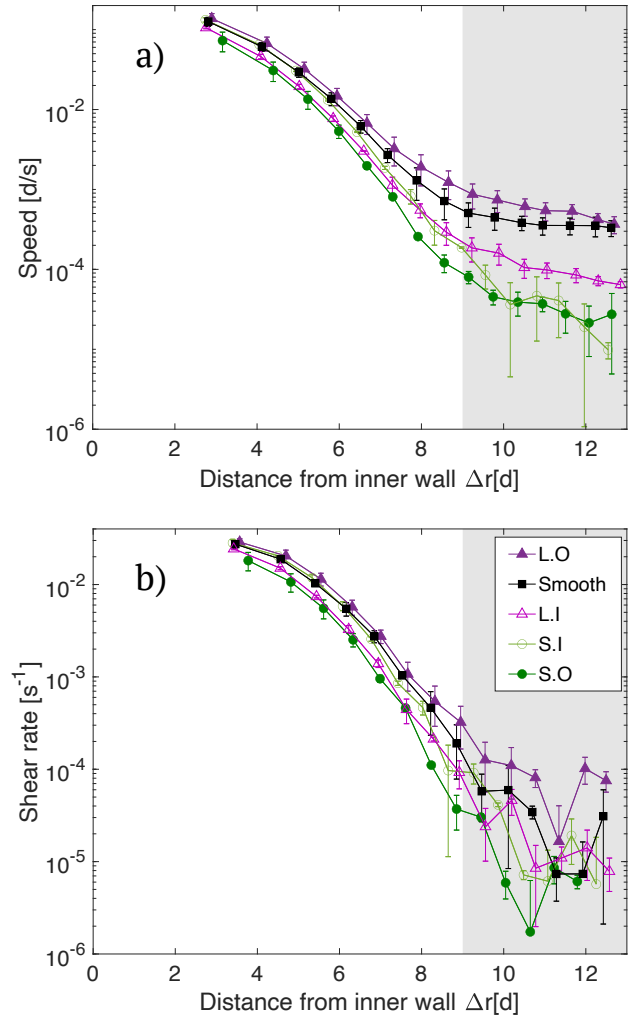

Figure 4. (a) Speed and (b) shear rate profiles, for boundaries with 5 different roughnesses. Gray region approximately corresponds to the nonlocal (creeping) region of the flow.

particles) can more precisely set a boundary condition, but are more sensitive to room humidity and temperature. This arises due the humidity and temperature influencing particles' interparticle friction and weak adhesion, both of which affect $\tau_{i}$. In the following, we report the boundary's influence on velocity and shear rate profiles at constant $\phi$, and shear and normal stress at constant $\tau_{i}$.

\subsection{Velocity and shear rate measurements}

For five of the six boundaries shown in Fig. 1, we obtain both the velocity profile $v(r)$ and the shear rate profile $\dot{\gamma}(r)$.
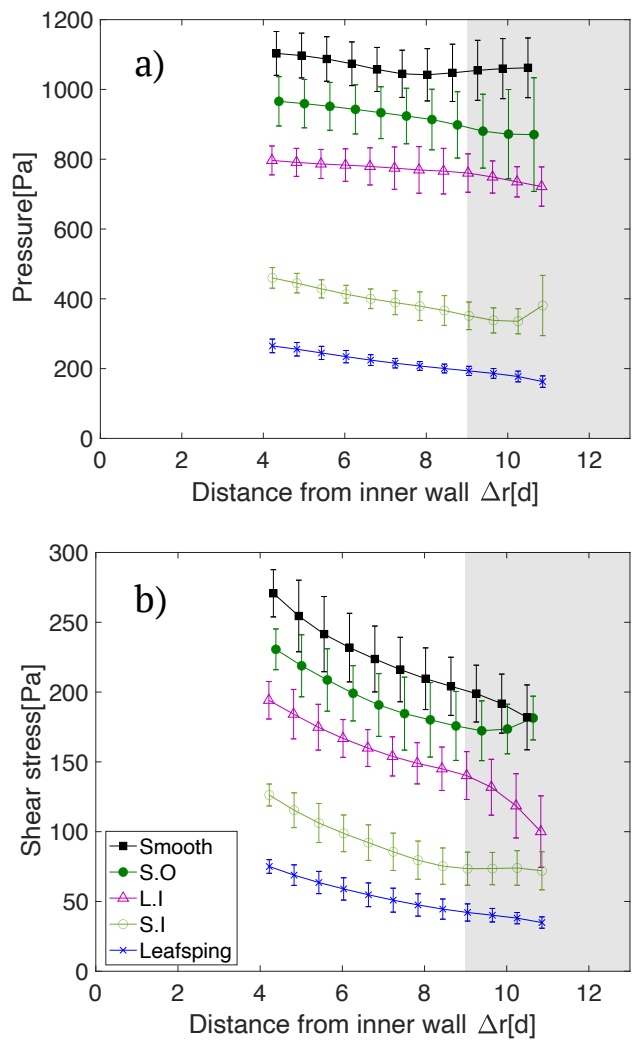

Figure 5. (a) Normal and (b) shear stress profiles for different roughness/compliance. Gray region approximately corresponds to the nonlocal (creeping) region of the flow.

Velocity profiles are calculated by particle tracking [17]. The shear rate profiles are obtained from derivatives of the speed profiles; due to our cylindrical geometry, this is $\dot{\gamma}(r)=\frac{1}{2}\left(\frac{\partial v}{\partial r}-\frac{v}{r}\right)$.

Fig. 4 shows the results, averaged over two independent trials $\left(10^{4}\right.$ frames, duration 1.5 hours, starting from fresh initial conditions) for each wall roughness, at constant $\phi$. We observe that the fastest-flowing portions of the flow are largely insensitive to the outer wall boundary. However, as the speed (or shear rate) drops by two orders of magnitude moving outwards, the nonlocal effects of the 
outer wall become increasingly apparent in the mismatch of the five datasets. This difference is more apparent in speed $(a)$ than in shear rate measurements $(b)$. We observe that the smoother the outer wall (smooth and large outies), the more slip is present at the outer wall. This is as expected, as roughness suppresses slip. However, we observe that the length scale of the roughness also plays a significant role. Roughness of the same depth but different width or inward/outward curvature provides different results. The walls with roughness of approximately equivalent depth and width (small innies and outies) are the best at suppressing wall slip, perhaps because they closely correspond to a continuation of the material. Interestingly, these also reduce the effects of shear-banding: the change in slope of the $v(r)$ curve is less pronounced between the local and nonlocal regions. This could arise due to stronger fluctuations induced by the roughness, betterfluidizing the nonlocal regime so that it does not move in a solid-body rotation.

\subsection{Stress measurements}

To quantify $P$ and $\tau$, we time-average 2000 frames of the coarse-grained stress field. For these experiments, measurements under five of the six roughness/compliance conditions were obtained at $\tau_{i}$-constant. The reported data does not extend all the way to the inner and outer walls due to lighting imperfections near the inner wall, as well as the coarse-graining length $w$ near both walls. Future work will complete the comparison with $\S 3.1$.

Fig. 5 shows the resulting stress profiles $P(r)$ and $\tau(r)$, calculated by averaging the stress $P$ and $\tau$ within concentric rings of width $0.56 \mathrm{~d}$. If there were no friction with the floor of the apparatus, the shear stress would follow the relation $\tau(r)=\tau\left(R_{i}\right)\left(\frac{R_{i}}{r}\right)^{2}$ and the pressure would be constant. However, the stress profiles are affected by basal friction and the softness/tilt of the photoelastic particles. As such, we observe $P(r)$ to be slightly decreasing instead of constant. The exceptions are the runs observed for the small innies and smooth boundaries: the pressure increases near the outer wall for different reasons. For the small innies boundary, the trapping of particles within individual indentations causes the increase in pressure: since the width of the indentations matches the particle diameter, some pressure is applied on particles from the outer wall. For the smooth boundary, particles form an ordered packing at the wall that may be related to the increased $\sigma_{\theta \theta}$ and $P$. The shear stress falls off away from the inner wall at approximately the same rate for all boundaries, but is strongly affected by the choice of boundary near the outer wall. Because the leafspring boundary, unlike the other boundaries, is deformable and does not maintain a constant volume, the stress is overall lower.

\section{Conclusions}

We have observed that boundary roughness strongly controls both the flow profile $v(r)$ and shear rate profile $\dot{\gamma}(r)$, particularly as measured at the outer wall. This is also the region of the flow most sensitive to nonlocal effects. Photoelastic techniques provide us full stress profile of the flow, allowing us to observe that the pressure $P$ and shear stress $\tau$, measured by photoelastic techniques, are similarly affected by the roughness and compliance of the wall. Future work will elucidate the nontrivial connection between these observations and the stress ratio $\mu(I)$, allowing us to separately determine constitutive parameters and boundary conditions.

Acknowledgements: This work was supported by the International Fine Particle Research Institute (IFPRI).

\section{References}

[1] Y. Forterre, O. Pouliquen, Annu. Rev. Fluid Mech. 40, 1 (2008)

[2] G. Koval, J.N. Roux, A. Corfdir, F. Chevoir, Phys. Rev. E 79, 021306 (2009)

[3] GDR MiDi, Eur. Phys. J. E 14, 341 (2004)

[4] X. Cheng, J.B. Lechman, A. Fernandez-Barbero, G.S. Grest, H.M. Jaeger, G.S. Karczmar, M.E. Möbius, S.R. Nagel, Phys. Rev. Lett. 96, 038001 (2006)

[5] K. Nichol, A. Zanin, R. Bastien, E. Wandersman, M. van Hecke, Phys. Rev. Lett. 104, 078302 (2010)

[6] K.A. Reddy, Y. Forterre, O. Pouliquen, Phys. Rev. Lett. 106, 108301 (2011)

[7] K. Kamrin, G. Koval, Phys. Rev. Lett. 108, 1 (2012)

[8] D.L. Henann, K. Kamrin, PNAS 110, 6730 (2013)

[9] M. Bouzid, A. Izzet, M. Trulsson, E. Clement, P. Claudin, B. Andreotti, Eur. Phys. J. E 38, 125 (2015)

[10] P.V. Dsouza, P.R. Nott, J. Fluid Mech. 888, R3 (2020)

[11] Z. Tang, T. Brzinski, M. Shearer, K.E. Daniels, Soft Matter 14, 3040 (2018)

[12] K.E. Daniels, J.E. Kollmer, J.G. Puckett, Rev. Sci. Instrum. 88, 051808 (2017)

[13] A. Abed Zadeh, J. Bares, T.A. Brzinski, K.E. Daniels, J. Dijksman, N. Docquier, H.O. Everitt, J.E. Kollmer, O. Lantsoght, D. Wang et al., Granul. Matter. 21, 83 (2019)

[14] J.E. Kollmer, Photo-Elastic Granular Solver (PEGS), https://github.com/jekollmer/PEGS

[15] K. Liu, J.E. Kollmer, K.E. Daniels, J. Schwarz, S. Henkes, Phys. Rev. Lett. 126, 088002 (2021)

[16] T. Weinhart, R. Hartkamp, A.R. Thornton, S. Luding, Phys. Fluids. 25, 070605 (2013)

[17] Matlab Particle Tracking, https://site. physics.georgetown.edu/matlab/ 